\title{
High-Sensitivity Cardiac Troponin T Is a Risk Factor for Major Adverse Cardiovascular Events and All-Cause Mortality: A 9.5-Year Follow-Up Study
}

\author{
Xiaona Wang, ${ }^{1}$ Peiqi Wang, ${ }^{2}$ Ruihua Cao, ${ }^{1}$ Xu Yang, ${ }^{1}$ Wenkai Xiao, ${ }^{1}$ Yun Zhang, \\ Li Sheng $\left(\mathbb{D},{ }^{1}\right.$ and Ping Ye $\mathbb{B}^{1}$ \\ ${ }^{1}$ Department of Geriatric Cardiology, The Second Medical Center and National Clinical Research Center for Geriatric Diseases, \\ Chinese PLA General Hospital, Beijing, China \\ ${ }^{2}$ Department of Anesthesiology, The First Medical Center, Chinese PLA General Hospital, Beijing, China
}

Correspondence should be addressed to Li Sheng; shengli301@163.com and Ping Ye; yeping301@sina.com

Received 26 November 2020; Accepted 17 August 2021; Published 26 August 2021

Academic Editor: Robert J. Chen

Copyright ( 2021 Xiaona Wang et al. This is an open access article distributed under the Creative Commons Attribution License, which permits unrestricted use, distribution, and reproduction in any medium, provided the original work is properly cited.

Background. The relationship between high-sensitivity cardiac troponin $\mathrm{T}$ (hs-cTnT) and different cardiovascular events has been observed in several large community studies, and the results have been controversial. However, there is currently no crosssectional or longitudinal follow-up study on hs-cTnT in the Chinese population. Methods. We analyzed the association of plasma hs-cTnT levels with major adverse cardiovascular events (MACEs) and all-cause mortality in 1325 subjects from a longitudinal follow-up community-based population in Beijing, China. Results. In the Cox proportional hazards models analysis, the risk of MACEs increased with the increase of hs-cTnT levels (HR, 1.223, 95\% CI, 1.054-1.418, $P=0.008$ ). Increased hs-cTnT levels were associated with coronary events (HR, 1.391, 95\% CI, 1.106-1.749, $P=0.005)$ in Model 4. Cox proportional risk regression model analysis revealed that increased hs-cTnT levels were associated with an increased risk of mortality (HR, 1.763, 95\% CI, 1.224-2.540, $P=0.002)$, even after adjusting hs-CRP and NT-proBNP. The area under the ROC curve for predicting MACEs was 0.559 (95\% CI, $0.523-0.595, P=0.001$ ). The areas under the ROC curve for predicting coronary events and mortality were 0.629 (95\% CI, 0.580-0.678, $P<0.001)$ and 0.644 (95\% CI, 0.564-0.725, $P<0.001)$, respectively. Conclusions. Our findings in the Chinese cohort support that hs-cTnT is a risk factor for major adverse cardiovascular events and all-cause mortality.

\section{Introduction}

It is extremely challenging to predict cardiovascular events in the general population; while the general population is unlikely to become a target of preventive measures, such measures have become a mainstay of how society addresses cardiovascular diseases [1]. Traditional risk factors such as hypertension, diabetes, hyperlipidemia, smoking, and obesity play important roles in the occurrence and development of cardiovascular disease. They can also be used as important prognostic factors for risk stratification and the prognostic evaluation of patients, but these traditional risk factors cannot be used to explain all prognostic risks. In recent years, new biomarkers, such as high-sensitivity cardiac troponin $\mathrm{T}$ (hs-cTnT) and nitrogen -terminated B-type brain key precursors (NT-proBNP), have been widely used in clinical practice and have been proven to be risk factors for cardiovascular diseases independent of traditional risk factors.

cTnT can be effectively used to discover subclinical heart disease and assess the risk of future cardiovascular disease, but the low detection rate of standard measurement methods limits its clinical application [2]. It is possible to measure cTnT with high sensitivity with the advancement of science and technology, and high-sensitivity cardiac troponin T (hscTnT) has emerged. Studies have found that the concentration of hs-cTnT in patients with stable coronary heart disease is significantly related to cardiovascular death or 
congestive heart failure $[3,4]$. Subsequently, the prognostic value of hs-cTnT in the general population was also confirmed. A study involving 4,221 elderly community residents with a median follow-up time of 11.8 years found that baseline hs-cTnT and the change of hs-cTnT were significantly related to the incidence of heart failure and cardiovascular death [5]. Since then, the relationship between hscTnT and different cardiovascular events has been observed in several large community studies, and the results have been controversial [5-7]. New research has also shown differences in hs-cTnT levels among different ethnic groups. However, there is currently no cross-sectional or longitudinal followup study on hs-cTnT in the Chinese population.

Therefore, the current study examined the relationship of hs-cTnT with mortality and cardiovascular events by investigating a community population without the definite cardiovascular disease to ascertain the following: (1) the predictive relationship between the hs-cTnT level and mortality and cardiovascular events and (2) the predictive relationship between the change in hs-cTnT level and mortality and cardiovascular events in a large communitybased longitudinal sample from China.

\section{Methods}

2.1. Subjects. After a routine health check-up between September 2007 and January 2009, a total of 1,680 subjects were initially eligible for cross-sectional analysis. During the follow-up from February 2013 to September 2013, 181 people were lost to follow-up due to various reasons. Finally, 1,499 subjects with complete data were included in the 5year follow-up analysis (follow-up rate $89.2 \%$ ). During the follow-up from June 2017 to September 2018, 174 people were lost to follow-up, and eventually, 1,325 subjects completed the follow-up (follow-up rate $89.2 \%$ ). The research protocol of this project was approved by the ethics committee of the General Hospital of the PLA, and each subject provided informed written consent. The median follow-up interval for the original subjects was 9.5 years. During these visits, all participants received a questionnaire survey. Physical examinations, biochemical indicators, and biomarkers (including hs-cTnT) were reviewed at the same time. Any event reported was subject to verification through medical records, death certificates, pathological autopsy results, and objective coronary angiographic examination results and were jointly judged by two clinically experienced doctors.

2.2. Clinical Data Collection. Height $(\mathrm{cm})$ and weight $(\mathrm{kg})$ were measured. Systolic and diastolic blood pressures (SBP and DBP) were measured in the right arm twice in a sitting position after $5 \mathrm{~min}$ of rest. Blood samples were collected from participants after an overnight fast. Concentrations of fasting blood glucose (FBG), total cholesterol (TC), triglycerides (TGs), low-density lipoprotein cholesterol (LDL$\mathrm{C})$, high-density lipoprotein cholesterol (HDL-C), homocysteine (Hcy), and uric acid (UA) were detected on Roche Diagnostics GmbH (Roche Diagnostics GmbH, Mannheim,
Germany). Serum creatinine (CR) was determined by a Roche enzyme assay kit on the Hitachi 7600 automatic analyzer (Hitachi, Tokyo, Japan). High-sensitivity C-reactive protein (hs-CRP) was detected on a Diension RxL Max analyzer (Siemens Healthcare Diagnostic) using an immunoassay method kit (Siemens Healthcare Diagnostic, USA, IN). N-Terminal B-type brain natriuretic peptide (NTproBNP) was detected on an autoanalyzer using a Roche Diagnostics GmbH (Roche Diagnostics GmbH). Highsensitivity cardiac troponin $\mathrm{T}$ (hs-cTnT) was measured using the high-sensitivity Troponin T Elecsys kit (Roche Diagnostics $\mathrm{GmbH}$, Mannheim, Germany). The concentration unit of hs-cTnT is $\mathrm{pg} / \mathrm{mL}$, and the coefficient of variation between batches is $8 \%$ at $10 \mathrm{pg} / \mathrm{mL}$ and $2.5 \%$ at $100 \mathrm{pg} / \mathrm{mL}$ [3].

2.3. Definition of Variables. Body mass index (BMI): weight $(\mathrm{kg}) /$ height $^{2}\left(\mathrm{~m}^{2}\right)$.

Hypertension: SBP $\geq 140 \mathrm{mmHg}$ and/or DBP $90 \mathrm{mmHg}$ or those who have taken antihypertensive drugs.

Diabetes mellitus: fasting venous blood glucose $\geq 7.0 \mathrm{mmol} / \mathrm{L}$, oral glucose tolerance test (OGTT) with $2 \mathrm{~h}$ blood glucose $\geq 11.1 \mathrm{mmol} / \mathrm{L}$, symptoms of hyperglycemia and random blood glucose $\geq 11.1 \mathrm{mmol} / \mathrm{L}$, or receiving hypoglycemic treatment [8].

Estimated glomerular filtration rate (eGFR): $141 \times \mathrm{min}$ $(\mathrm{Scr} / \mathrm{k}, 1) \alpha \times \max (\mathrm{Scr} / \mathrm{k}, 1)-1.209 \times 0.993$ age $\times 1.018$ (if female) $\times 1.159$ (if black), where Scr is plasma creatinine $(\mathrm{mg} /$ $\mathrm{dL}), k$ is 0.7 for females and 0.9 for males, $\alpha$ is -0.329 for females and -0.411 for males, min indicates the minimum of $\mathrm{Scr} / \mathrm{k}$ or 1 , and max indicates the maximum of $\mathrm{Scr} / \mathrm{k}$ or 1 .

hs-cTnT: the lowest detectable concentration according to the kit instructions is $3 \mathrm{pg} / \mathrm{mL}$ (according to the reagent instructions), which is used as the cut-off value in this study. Therefore, hs-cTNT levels $\geq 3 \mathrm{pg} / \mathrm{mL}$ are considered measurable. The 99th percentile for the hs-cTnT of a group of healthy people aged 20 to 70 years was $14 \mathrm{pg} / \mathrm{mL}$ [9], and hscTnT concentrations $\geq 14 \mathrm{pg} / \mathrm{mL}$ were generally considered to be elevated.

All-cause mortality was determined by a review of death certificates. The definition of MACEs comprised nonfatal myocardial infarction, newly diagnosed CHD (identified by coronary artery imaging or receiving coronary revascularization), stroke (ischemic or hemorrhagic), and cardiovascular mortality.

Cardiovascular death was defined as deaths related to atherosclerotic heart disease (fatal myocardial infarction and definite fatal coronary heart disease), cerebrovascular disease deaths (fatal stroke), and causes including heart failure death from other atherosclerotic and cardiovascular diseases [10].

MACEs were defined as cardiovascular death, nonfatal myocardial infarction, coronary revascularization, coronary heart disease, and stroke confirmed by coronary imaging [11].

Coronary heart disease (CHD) events were defined as coronary heart disease death, nonfatal myocardial infarction, coronary revascularization, and coronary heart disease diagnosed by coronary imaging. 
Stroke was defined as acute, focal damage to the central nervous system caused by vascular causes, which results in neurological deficits, including cerebral infarction (rather than internal hemorrhage) and subarachnoid hemorrhage [12].

2.4. Statistical Analyses. Continuous variables are expressed as the mean or median (interquartile range) \pm standard deviation (SD) and dichotomous variables are expressed as percentages. Analysis of continuous variables was performed by the $t$-test, and analysis of categorical variables was performed by $\chi^{2}$ test.

Pearson regression analysis and stepwise multivariate linear regression analysis were performed to evaluate the associations between baseline hs-cTnT and baseline traditional cardiovascular risk factors.

For the analyses of hs-cTnT as a categorical variable, subjects were divided into three groups according to their baseline hs-cTnT level: hs-cTnT $<3 \mathrm{pg} / \mathrm{mL}$, hs-cTnT between 3 and $14 \mathrm{pg} / \mathrm{mL}$, and hs-cTnT $\geq 14 \mathrm{pg} / \mathrm{mL}$. The relationship between baseline hs-cTnT levels and MACEs and coronary events and mortality was analyzed by Cox proportional hazards models. Models were defined as follows: model $1=$ adjusted for age and gender; model $2=$ adjusted for model $1+$ presence of hypertension or diabetes mellitus, current smoking status, SBP, postprandial blood glucose, TC, HDL-C, antihypertensive medication use, and antidiabetic medication use; model $3=$ adjusted for model $2+$ eGFR; model $4=$ adjusted for model $3+$ hs-CRP and NTproBNP (both after logarithmic transformation).

We also analyzed the relationship between hs-c $\mathrm{TnT}$ as a continuous variable and endpoints, in which values of cTnT that were below the detection limit were assigned to $1.5 \mathrm{ng} / \mathrm{L}$ (i.e., one-half of the lower limit of detection).

SPSS17.0 software was used for all statistical analyses. $P$ value $<0.05$ was considered statistically significant.

\section{Results}

3.1. General Characteristics of the Study Population. The general characteristics of the population are shown in Table 1 . In this population, the average age was $59.10 \pm 9.8$ years, and $51.5 \%$ were females. Of these 1,325 people, 736 had a detectable level of hs-cTnT $(>3.0 \mathrm{pg} / \mathrm{ml})$, accounting for $55.54 \%$ of the total, as shown in Table 1 . The distribution range of hs-cTnT was from $3.03 \mathrm{pg} / \mathrm{ml}$ to $176.4 \mathrm{pg} / \mathrm{ml}$, and the median was $7.36 \mathrm{pg} / \mathrm{ml}(25 \%$ and $75 \%$ digits were $4.76 \mathrm{pg} / \mathrm{ml}$ and $15.58 \mathrm{pg} / \mathrm{ml}$, respectively). There were 143 people with an increased hs-cTnT ( $\geq 14.0 \mathrm{pg} / \mathrm{ml})$, accounting for $10.79 \%$ of the total. Comparing the three groups, the cardiovascular risk factors in the middle and increased groups were significantly higher than those in the lower group. In addition, cardiovascular risk factors, such as being male, history of diabetes, history of hypertension, FBG, uric acid, Hcy, in the increased group were significantly different from those in the other two groups.

3.2. Clinical Factors Affecting Hs-cTnT. At baseline, univariate analysis showed that elderly $(r=0.085, P=0.004)$, male $(r=0.120, P<0.001)$, smoking $(r=0.110, P=0.001)$, hypertension $\quad(r=0.278, \quad P<0.001), \quad$ Scr $\quad(r=0.101$, $P=0.001)$, FBG $(r=0.065, P=0.026)$, BNP $(r=0.119$, $P<0.001)$, and hs-CRP $(r=0.087, P=0.005)$ were positively correlated with hs-cTnT; eGFR $(r=-0.091, P=0.002)$ was negatively correlated with hs-cTnT. Multivariate linear regression analysis showed that male $(\beta=0.725, P<0.001)$, hypertension $(\beta=0.883, \quad P<0.001), \quad$ FBG $\quad(\beta=0.064$, $P<0.001)$, TC $(\beta=0.227, P<0.001)$, and LDL-C $(\beta=0.222$, $P=0.003)$ were positively correlated with hs-cTnT, while eGFR $(\beta=-0.596, P<0.001)$ was negatively correlated with hs-cTnT (Table 2).

3.3. Associations of Baseline Hs-cTnT Levels with Major Adverse Cardiovascular Events (MACEs), Coronary Events, and Mortality. During the follow-up period, a total of 191 participants experienced MACEs, and the incidence increased from $11.05 \%$ in the lowest (hs-cTnT $<3 \mathrm{pg} / \mathrm{ml}$ ) group to $23.02 \%$ in the highest group (hs-cTnT $>14 \mathrm{pg} / \mathrm{ml}$ ), as demonstrated by Kaplan-Meier survival analysis $(P<0.001)$ (Figure $1(\mathrm{a}))$. In addition, after adjusting for multiple factors in the Cox proportional hazards models analysis (Model 4), the risk of MACEs increased with the increase of hs-cTnT levels (HR, 1.223, 95\% CI, 1.054-1.418, $P=0.008$ ) (Table 3).

Similar trends were discovered for coronary events. A total of 121 participants experienced coronary events, and the risk for coronary events and all-cause mortality by baseline hs-cTnT level is shown in Figure 1(b). Increased hscTnT levels were associated with coronary events (HR, 1.391, 95\% CI, 1.106-1.749, $P=0.005$ ) in Model 4 (Table 3).

During the median follow-up of 9.5 years, a total of 84 deaths occurred, and the mortality rate increased significantly from $8.08 \%$ in the lowest quartile group (hs-cTnT $<3 \mathrm{pg} / \mathrm{mL}$ ) to $10.32 \%$ in the highest quartile (hs-cTnT $>14 \mathrm{pg} / \mathrm{mL})(P<0.01)$ (Figure $1(\mathrm{c}))$. After adjusting for age, gender, blood pressure, blood lipids, renal function, and other traditional cardiovascular risk factors, Cox proportional risk regression model analysis revealed that increased hs-cTnT levels were associated with an increased risk of mortality (HR, 1.763, 95\% CI, 1.224-2.540, $P=0.002)$, even after adjusting hs-CRP and NT-proBNP (Model 4) (Table 3).

3.4. Hs-cTnT Predicts MACEs and Mortality. The ROC curve was used to analyze the accuracy of hs-cTnT in predicting MACEs (Figures 2(a)-2(c)). The area under the ROC curve for predicting MACEs was 0.559 (95\% CI, 0.523-0.595, $P=0.001$ ) (Figure 2(a)). The best cut-off value of hs-cTnT for predicting MACEs was $5.01 \mathrm{pg} / \mathrm{mL}$. At the same time, the sensitivity and specificity were $53.8 \%$ and 59.1\%, respectively.

The areas under the ROC curve for predicting coronary events and mortality were 0.629 (95\% CI, $0.580-0.678$, $P<0.001$ ) (Figure 2(b)) and 0.644 (95\% CI, 0.564-0.725, $P<0.001$ ) (Figure 2(c)), respectively. The best cut-off value of hs-cTnT for predicting coronary events and mortality were $4.05 \mathrm{pg} / \mathrm{mL}$ and $4.6 \mathrm{pg} / \mathrm{mL}$, respectively. When the hscTnT value was $4.05 \mathrm{pg} / \mathrm{mL}$, the sensitivity for predicting 
TABLE 1: Baseline characteristics and laboratory test results of subjects.

\begin{tabular}{|c|c|c|c|c|}
\hline & \multicolumn{4}{|c|}{ Hs-TnT group (ng/L) } \\
\hline & Group $1(n=589)$ & Group $2(n=593)$ & Group $3(n=143)$ & $P$ value \\
\hline Age (year) & $58.4 \pm 12.3$ & $60.7 \pm 11.7$ & $62.7 \pm 11.1$ & 0.010 \\
\hline Male $(n(\%))$ & $155(26.31 \%)$ & $308(51.94 \%)$ & $89(62.2 \%)$ & 0.001 \\
\hline BMI $\left(\mathrm{kg} / \mathrm{m}^{2}\right)$ & $25.4 \pm 3.8$ & $25.5 \pm 3.6$ & $25.6 \pm 3.7$ & 0.872 \\
\hline Smoking (n (\%)) & $51(8.66 \%)$ & $108(18.21 \%)$ & $30(20.97 \%)$ & 0.022 \\
\hline $\mathrm{SBP}(\mathrm{mmHg})$ & $131.8 \pm 15.6$ & $132.7 \pm 17.4$ & $134.7 \pm 16.5$ & 0.893 \\
\hline $\mathrm{DBP}(\mathrm{mmHg})$ & $77.2 \pm 12.6$ & $77.1 \pm 11.7$ & $77.6 \pm 12.5$ & 0.275 \\
\hline $\mathrm{TC}(\mathrm{mmol} / \mathrm{L})$ & $5.05 \pm 0.91$ & $5.03 \pm 0.93$ & $4.89 \pm 0.92$ & 0.259 \\
\hline TG $(\mathrm{mmol} / \mathrm{L})$ & $1.92 \pm 1.69$ & $1.76 \pm 1.09$ & $2.07 \pm 1.83$ & 0.018 \\
\hline HDL-C (mmol/L) & $1.34 \pm 0.45$ & $1.38 \pm 0.36$ & $1.33 \pm 0.32$ & 0.298 \\
\hline LDL-C (mmol/L) & $2.96 \pm 0.75$ & $2.92 \pm 0.71$ & $2.86 \pm 0.74$ & 0.512 \\
\hline $\mathrm{FBG}(\mathrm{mmol} / \mathrm{L})$ & $5.63 \pm 2.24$ & $5.29 \pm 1.46$ & $5.85 \pm 2.22$ & $<0.001$ \\
\hline $\operatorname{Scr}(\mathrm{mmol} / \mathrm{L})$ & $67.61 \pm 15.91$ & $66.27 \pm 16.48$ & $73.49 \pm 20.29$ & $<0.001$ \\
\hline $\mathrm{eGFR}\left(\mathrm{ml} / \mathrm{min}^{-1} / 1.73 \mathrm{~m}^{2}\right)$ & $90.57 \pm 14.83$ & $87.38 \pm 13.73$ & $85.14 \pm 13.51$ & $<0.001$ \\
\hline hs-CRP (mg/L) & $2.4(1.3,3.4)$ & $2.3(1.4,3.4)$ & $2.4(1.6,3.6)$ & 0.365 \\
\hline $\mathrm{BNP}$ & $35.51 \pm 15.03$ & $38.32 \pm 10.95$ & $48.55 \pm 18.37$ & $<0.001$ \\
\hline Hs-TnT (pg/mL) & & $5.24 \pm 2.79$ & $26.06 \pm 17.94$ & $<0.001$ \\
\hline
\end{tabular}

TC, total cholesterol; HDL-C, high-density lipoprotein cholesterol; TG, triglyceride; LDL-C, low-density lipoprotein cholesterol; SBP, systolic blood pressure; DBP, diastolic blood pressure; BMI, body mass index; FBG, fast blood glucose; eGFR, estimated glomerular filtration rate; HR, heart rate; Scr, serum creatinine; hs-CRP, high-sensitive C-reactive protein; BNP, brain natriuretic peptide; Hs-TnT, high-sensitivity troponin T.

TABle 2: Clinical factors affecting Hs-TnT.

\begin{tabular}{lcccc}
\hline All subjects $(n=1325)$ & Hs-TnT & & Hs-TnT \\
\hline Age & $r$ & $P$ value & $\beta$ & $0.002 \sim 0.007$ \\
Male & 0.085 & 0.004 & 0.002 & 0.308 \\
Smoking & 0.120 & $<0.001$ & 0.725 & $0.340 \sim 1.110$ \\
Diabetes & 0.110 & 0.001 & 0.320 & $-0.086 \sim 0.725$ \\
Hypertension & 0.144 & $<0.001$ & 0.232 & $0.058 \sim 0.522$ \\
TG* & 0.278 & $<0.001$ & 0.883 & $0.501 \sim 1.265$ \\
HDL-C* & 0.013 & 0.646 & 0.044 & $-0.002 \sim 0.091$ \\
LDL-C & 0.029 & 0.321 & $-0.070 \sim 0.270$ \\
TC & 0.019 & 0.515 & 0.100 & $0.077 \sim 0.367$ \\
SBP & 0.049 & 0.092 & 0.222 & $0.102 \sim 0.351$ \\
DBP & 0.011 & 0.702 & 0.227 & $0.033 \sim 0.060$ \\
BMI & 0.049 & 0.093 & 0.046 & $0.000 \sim 0.003$ \\
FBG & 0.033 & 0.258 & 0.002 & 0.003 \\
Cr & 0.065 & 0.026 & 0.010 & $0.002 \sim 0.007$ \\
eGFR* & 0.101 & 0.001 & 0.064 & $0.037 \sim 0.091$ \\
hs-CRP & -0.091 & 0.002 & 0.231 & 0.072 \\
BNP & 0.087 & 0.005 & -0.596 & $0.098 \sim 0.391$ \\
TC & 0.119 & $<0.001$ & 0.044 & $-0.915 \sim-0.277$ \\
\end{tabular}

TC, total cholesterol; HDL-C, high-density lipoprotein cholesterol; TG, triglyceride; LDL-C, low-density lipoprotein cholesterol; SBP, systolic blood pressure; DBP, diastolic blood pressure; BMI, body mass index; FBG, fast blood glucose; eGFR, estimated glomerular filtration rate; HR, heart rate; Scr, serum creatinine; hs-CRP, high-sensitive C-reactive protein; BNP, brain natriuretic peptide; Hs-TnT, high-sensitivity troponin T. ${ }^{*}$ Natural logarithm transformed. $\$$ Covariates in the multiple-adjusted models included age, gender, hypertension, diabetes, current smoking, and levels of plasma.

coronary events was $72.5 \%$, and the specificity was $49.8 \%$. When the hs-cTnT value was $4.6 \mathrm{pg} / \mathrm{mL}$, the sensitivity for predicting mortality was $74.1 \%$, and the specificity was $51.4 \%$.

\section{Discussion}

To our knowledge, this study is the first follow-up study to evaluate the predictive value of hs-cTnT for cardiovascular events and mortality conducted in China. Through the follow-up study of this community population for nearly 10 years, this study has some significance. First, we observed minor subclinical myocardial injury in the general population using the latest generation of hs-cTnT measurement methods, rather than only focusing on the elderly or highrisk population. We found that the hs-cTnT detection rate in this population was $54.70 \%$. Second, the results confirmed that the baseline level of hs-cTnT can predict the mortality and cardiovascular event risk of the population of the community. Furthermore, we also found that increases in hs-cTnT also have predictive value for cardiovascular events and mortality. 


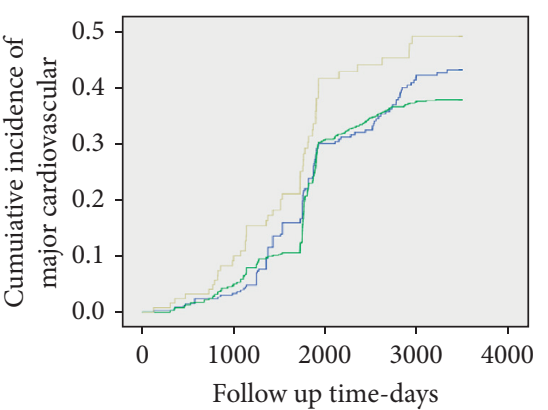

Hs-TnT category

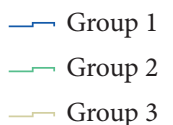

(a)

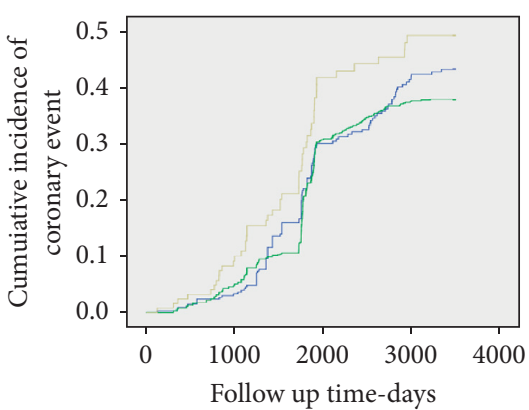

Hs-TnT category

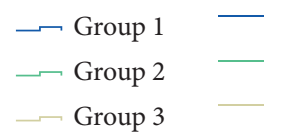

(b)

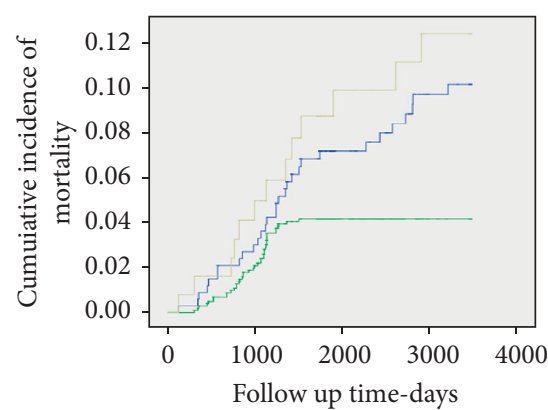

Hs-TnT category

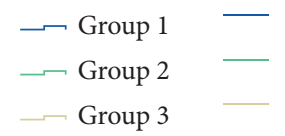

(c)

FIGURE 1: Risk for cardiovascular events and all-cause mortality by baseline hs-cTnT level. Kaplan-Meier survival curves indicating cumulative incidence of major adverse cardiovascular events (a), coronary event (b), and all-cause mortality (c) across baseline hs-cTnT categories.

TABle 3: Cox proportional hazards models analysis for associations between baseline hs-cTnT levels and outcomes.

\begin{tabular}{lcccccc}
\hline & \multicolumn{2}{c}{ Mace } & \multicolumn{2}{c}{ Coronary event } & \multicolumn{2}{c}{ All-cause mortality } \\
& HR $(95 \% \mathrm{CI})$ & $P$ value & HR $(95 \% \mathrm{CI})$ & $P$ value & HR $(95 \% \mathrm{CI})$ & $P$ value \\
\hline Unjust & $1.313(1.144-1.506)$ & $<0.001$ & $1.747(1.440-2.210)$ & $<0.001$ & $1.996(1.470-2.709)$ & $<0.001$ \\
Model1 & $1.188(1.026-1.375)$ & 0.021 & $1.395(1.122-1.734)$ & 0.003 & $1.472(1.025-2.115)$ & 0.036 \\
Model2 & $1.183(1.016-1.377)$ & 0.030 & $1.392(1.110-1.746)$ & 0.004 & $1.657(1.145-2.397)$ & 0.007 \\
Model3 & $1.223(1.054-1.418)$ & 0.008 & $1.391(1.106-1.749)$ & 0.005 & $1.763(1.224-2.540)$ & 0.002 \\
\hline
\end{tabular}

Models are defined as follows: model 1 = adjusted for age and gender; model 2 = adjusted for model $1+$ presence of hypertension or diabetes mellitus, current smoking status, systolic blood pressure, postprandial blood glucose, total cholesterol, high-density lipoprotein cholesterol, antihypertensive medication use, and antidiabetic medication use; model $3=$ adjusted for model $2+$ estimated glomerular filtration rate; model $4=$ adjusted for model $3+$ high-sensitivity $\mathrm{C}$ reactive protein and $\mathrm{N}$-terminal pro-B-type natriuretic peptide (both after logarithmic transformation). MACE, major adverse cardiovascular event.

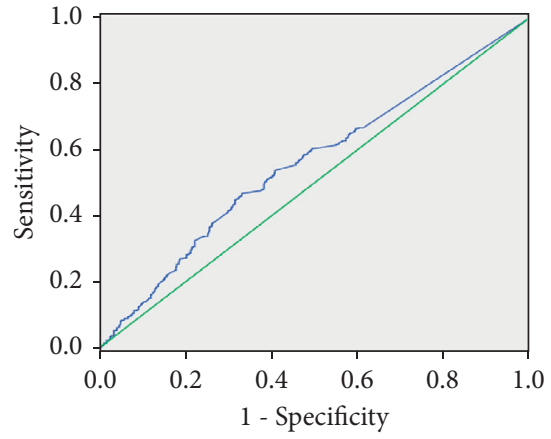

(a)

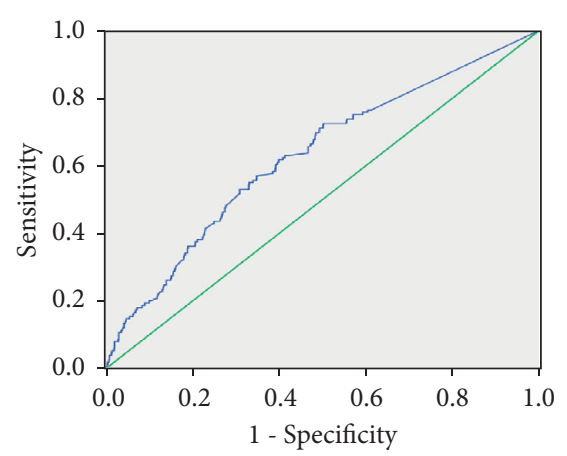

(b)

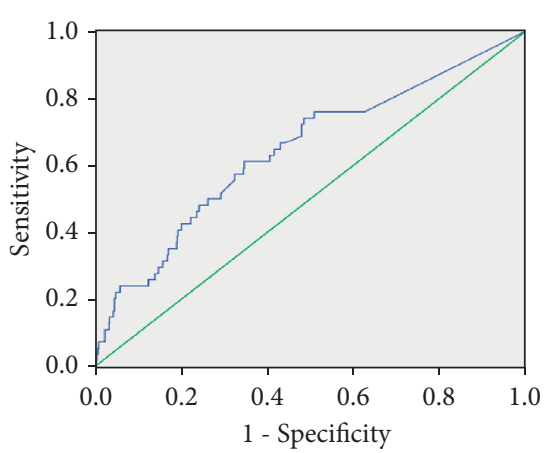

(c)

Figure 2: Hs-TnT predicts the ROC curve of major adverse cardiovascular events (a), coronary event (b), and all-cause mortality (c). The area under the curve (AUC) is (a) 0.559 (95\% confidence interval: $0.523-0.595, P=0.001$ ); (b) 0.629 (95\% confidence interval: $0.580-0.678$, $P<0.001$ ); and (c) 0.644 (95\% confidence interval: $0.564-0.725, P<0.001$ ), respectively.

In recent years, highly sensitive methods for detecting hs-cTnT have increased in clinical practice. At present, there is not a very clear definition of hs-cTnT, which is mainly based on the analytical performance of the lowest detection limit and the measurement precision in the low cTnT concentration range. The definition of hs-cTnT is as follows [13-15]: high-sensitivity methods can detect cTnT (e.g., as low as $10 \mathrm{ng} / \mathrm{L}$ ) levels that cannot be detected by current traditional methods; the cTnT with the minimum detection value of $\mathrm{CV} \leq 10 \%$ and the 99th percentile value detected by the system or reagent that meets the requirements of the guidelines; or the cTnT can be detected in some or all of the surface healthy people and the 99th percentile value/ $\mathrm{CV} \leq 10 \%$. In 2018, the International Federation of Clinical 
Chemistry and Laboratory Medicine released the latest specific quality standards for troponin detection [16], which proposed that high-sensitivity troponin should meet the detection rate of more than $50 \%$ in apparently healthy males and females. New research has also shown differences in hscTnT levels among different ethnic groups [17, 18]. The results of this study suggest that the prevalence of hs-cTnT levels $>14.0 \mathrm{pg} / \mathrm{mL}$ was approximately $11.0 \%$, which is slightly higher than the previous studies. In the current study, the detection rate of hs-cTnT in the general population in the Beijing community was $70.65 \%$ for males and $43.85 \%$ for females, respectively, which was similar to foreign studies. Our findings are slightly different from the previous study, probably because of the middle-aged and elderly in the study. Although there is no clear history of cardiovascular disease, traditional cardiovascular risk factors, such as hypertension, diabetes, and smoking, are still common in this group of middle-aged and elderly people. Undiagnosed resting myocardial ischemia and chronic changes in cardiac structure and function may still possibly be present.

hs-cTnT has been recognized as the first marker of myocardial injury in the diagnosis, risk stratification, and prognosis of an acute coronary syndrome (ACS), and its value has been recognized at home and abroad [19]. Gareth et al. concluded that early elevation in plasma hs-cTnT within $24 \mathrm{~h}$ of elective noncardiac surgery precedes the subsequent development of noncardiac organ dysfunction [20]. Because of its high sensitivity and specificity, hs-cTnT has been agreed upon by the European Heart Association and Chinese experts as the best basis for early diagnosis of acute myocardial infarction (AMI) and risk stratification of heart diseases [21]. In predicting future events, multiple trials have confirmed an association between elevated hscTnT levels and mortality or cardiovascular death in some populations at high risk for cardiovascular disease. In addition, few studies have reported the association of hs-cTnT levels with cardiovascular events in individuals from a general population, and the results have been controversial. The Atherosclerosis Risk in Communities (ARIC) Study found an association between detectable cTnT and a highly sensitive assay was associated with incident CHD, mortality, and $\mathrm{HF}$ in individuals without known $\mathrm{CHD} /$ stroke [22]. Evidence from a prospective study of the cTnT detected with a highly sensitive assay supports a stronger association with structural heart disease and subsequent risk for all-cause mortality [6]. Changes in cTnT levels measured with a highly sensitive assay were found to be significantly associated with incident $\mathrm{HF}$ and cardiovascular death [7]. Welsh did not identify a significant relationship between baseline cTnT levels and some CVD outcomes, whereas they reported that cTnT is more strongly associated with the risk of non-CVD death [23]. Although hs-cTnT as a continuous variable is significantly related to MACEs and mortality, when used as a categorical variable, the clear correlation between hs-cTnT and mortality was only present in the highest group (>14 pg/ $\mathrm{mL}$ ). In the Cox proportional hazard model analysis, the correlation between hs-cTnT and MACEs and mortality did not decrease significantly after adjusting for traditional risk factors, renal function, and hs-CRP. However, when the NTproBNP level was further adjusted, the risk of MACEs and mortality was significantly reduced, suggesting that NTproBNP and hs-cTnT partially overlap information about abnormalities in cardiac structure and function.

Few studies have reported the optimal hs-cTnT cut-off for predicting MACEs and mortality in the general population. The use of an equivalent cut-off for hs-cTnT merits further discussion, and universally using a cut-off of $0.014 \mu \mathrm{g} / \mathrm{L}$ may result in the improper diagnosis of acute CVD [24]. An individual patient data meta-analysis reported that the optimal cut-off value for all-cause death was $18 \mathrm{ng} / \mathrm{L}$ in a chronic heart failure population, whereas the optimal cut-off values increased progressively with worse renal function [25]. In our study, the ROC curve analysis predicted the area under the ROC curve for MACEs and mortality was 0.559 and 0.644 , respectively. When the hscTnT value was $5.01 \mathrm{pg} / \mathrm{mL}$, the sensitivity for predicting MACEs was $53.8 \%$ and the specificity was $59.1 \%$. When the hs-cTnT value was $4.6 \mathrm{pg} / \mathrm{mL}$, the sensitivity for predicting MACEs was $74.1 \%$ and the specificity was $51.4 \%$. Obviously, specialized research should determine whether patient management guided by the same cut-off value is beneficial to prognosis and cost-effective.

The mechanisms of small elevations of hs-cTnT in apparently healthy subjects are not fully understood. It is well known that serum elevation of cTnT is associated with myocardial ischemia, and a positive correlation between elevated hs-cTnT levels and new or suspected ECG ST-T changes or new left bundle branch block was recently described [4]. However, the study found that concentrations of hs-cTnT remained unchanged after exercise in patients with and without detectable ischemia [26], supporting that factors (e.g., coronary microvascular dysfunction [27], apoptosis [28, 29], or subclinical abnormalities of cardiac structure or function [30] could induce troponin release) in addition to ischemia contribute significantly to the risk associated with cTnT. Therefore, there may be several mechanisms underlying elevated troponin levels, and it is unclear which mechanisms are related to the outcomes observed in our study.

A new study shows that incorporating hs-cTnI testing into risk algorithms for patients with arteriosclerotic cardiovascular disease (ASCVD) provides enhanced risk stratification and leads to the reclassification of about $12 \%$ of patients into a more appropriate risk group [31]. Troponin levels should not be viewed just as a marker for myocardial injury and diagnosis of $\mathrm{MI}$ in acute coronary syndrome but should be used more frequently for assessing CVD risk in stable patients with ischemic heart disease. It is very meaningful to screen hs-cTnT in the general population. Elevated hs-cTnT levels in community populations may be an early warning device to reflect the risk of chronic cardiovascular disease or future cardiovascular disease. However, the increase of sensitivity may also lead to the decrease of diagnostic specificity, especially in diabetes, chronic kidney disease, or elderly men, where an increase of the hscTnT level is very common. Therefore, it is necessary to establish appropriate hs-cTnT diagnostic thresholds 
according to different populations. The deficiency of this study is the lack of objective examination to evaluate the structure and function of the heart, such as echocardiography and imaging examinations of coronary arteries.

\section{Conclusions}

Our findings in the Chinese cohort support that hs-cTnT is a risk factor for major adverse cardiovascular events and allcause mortality.

\section{Abbreviations}

hs-cTnT: High-sensitivity cardiac troponin T

SBP: $\quad$ Systolic blood pressures

DBP: $\quad$ Diastolic blood pressures

FBG: $\quad$ Fasting blood glucose

TC: $\quad$ Total cholesterol

TGs: $\quad$ Triglycerides

LDL-C: $\quad$ Low-density lipoprotein cholesterol

HDL-C: High-density lipoprotein cholesterol

Hcy: $\quad$ Homocysteine

hs-CRP: High-sensitivity C-reactive protein

NT-proBNP: N-Terminal B-type brain natriuretic peptide

MACEs: Major adverse cardiovascular events

ASCVD: $\quad$ Arteriosclerotic cardiovascular disease.

\section{Data Availability}

The data used to support the study are available from the corresponding author upon request.

\section{Ethical Approval}

This study was approved by the ethics committee of the People's Liberation Army General Hospital.

\section{Consent}

Each subject provided informed written consent.

\section{Disclosure}

This manuscript has been published in preprint, with no updates.

\section{Conflicts of Interest}

The authors declare that they have no conflicts of interest.

\section{Authors' Contributions}

$\mathrm{XW}$, LS, and PY designed the study; RC, XY, WX, and PW participated in the acquisition of data; XW and $\mathrm{PY}$ researched and evaluated the literature; XW performed the statistical analysis and wrote the first draft of the manuscript. All authors read and approved the final manuscript. Xiaona Wang and Peiqi Wang contributed equally to this study.

\section{Acknowledgments}

The authors thank colleagues at the Department of Laboratory Medicine, the PLA General Hospital, for help with biochemical measurements. The authors are also grateful to all study participants for their participation in the study. This research was supported by the Key Science and Technology Foundation of China (2020YFC2004805) to Dr. Sheng Li.

\section{References}

[1] U. N. Khot, M. B. Khot, C. T. Bajzer et al., "Prevalence of conventional risk factors in patients with coronary heart disease," Jama, vol. 290, no. 7, pp. 898-904, 2003, 2.

[2] M. Mueller, M. Vafaie, M. Biener, E. Giannitsis, and H. A. Katus, "Cardiac troponin T: from diagnosis of myocardial infarction to cardiovascular risk prediction," Circulation Journal, vol. 77, no. 7, pp. 1653-1661, 2013.

[3] T. Omland, J. A. de Lemos, M. S. Sabatine et al., "A sensitive cardiac troponin $\mathrm{T}$ assay in stable coronary artery disease," New England Journal of Medicine, vol. 361, no. 26, pp. 2538-2547, 2009.

[4] M. Král., D. Saňák, T. Veverka et al., "Troponin T in acute ischemic stroke," The American Journal of Cardiology, vol. 112, no. 1, pp. 117-121, 2013.

[5] C. R. deFilippi, J. A. de Lemos, R. H. Christenson et al., "Association of serial measures of cardiac troponin T using a sensitive assay with incident heart failure and cardiovascular mortality in older adults," Jama, vol. 304, no. 22, pp. 2494-2502, 2010.

[6] J. A. de Lemos, M. H. Drazner, T. Omland et al., "Association of troponin $\mathrm{T}$ detected with a highly sensitive assay and cardiac structure and mortality risk in the general population," Jama, vol. 304, no. 22, pp. 2503-2512, 2010.

[7] J. W. McEvoy, Y. Chen, C. E. Ndumele et al., "Six year change in high-sensitivity cardiac troponin $\mathrm{T}$ and risk of subsequent coronary heart disease, heart failure, and death," JAMA Cardiology, vol. 1, no. 5, pp. 519-528, 2016.

[8] D. B. Sacks, M. Arnold, G. L. Bakris et al., "Position statement executive summary: guidelines and recommendations for laboratory analysis in the diagnosis and management of diabetes mellitus," Diabetes Care, vol. 34, no. 6, pp. 1419-1423, 2011.

[9] E. Giannitsis, K. Kurz, K. Hallermayer, J. Jarausch, A. S. Jaffe, and H. A. Katus, "Analytical validation of a high-sensitivity cardiac troponin T assay," Clinical Chemistry, vol. 56, no. 2, pp. 254-261, 2010.

[10] D. G. Ives, A. L. Fitzpatrick, D. E. Bild et al., "Surveillance and ascertainment of cardiovascular events. The cardiovascular health study," Annals of Epidemiology, vol. 5, no. 4, pp. 278-285, 1995.

[11] T. J. Wang, P. Gona, M. G. Larson et al., "Multiple biomarkers for the prediction of first major cardiovascular events and death," New England Journal of Medicine, vol. 355, no. 25, pp. 2631-2639, 2006.

[12] B. G. Angeja, M. G. Shlipak, A. S. Go et al., "Hormone therapy and the risk of stroke after acute myocardial infarction in postmenopausal women," Journal of the American College of Cardiology, vol. 38, no. 5, pp. 1297-1301, 2001.

[13] V. C. Vasile, A. K. Saenger, J. M. Kroning, and A. S. Jaffe, "Biological and analytical variability of a novel high-sensitivity cardiac troponin T assay," Clinical Chemistry, vol. 56, no. 7, pp. 1086-1090, 2010. 
[14] F. S. Apple, P. O. Collinson, and IFCC Task Force on Clinical Applications of Cardiac Biomarkers, "Analytical characteristics of high-sensitivity cardiac troponin assays," Clinical Chemistry, vol. 58, no. 1, pp. 54-61, 2012.

[15] F. K. Korley and A. S. Jaffe, "Preparing the United States for high-sensitivity cardiac troponin assays," Journal of the American College of Cardiology, vol. 61, no. 17, pp. 1753-8, 2013.

[16] A. H. B. Wu, R. H. Christenson, D. N. Greene et al., "Clinical laboratory practice recommendations for the use of cardiac troponin in acute coronary syndrome: expert opinion from the academy of the American association for clinical Chemistry and the task force on clinical applications of cardiac bio-markers of the international federation of clinical Chemistry and laboratory medicine," Clinical Chemistry, vol. 64, no. 4, pp. 645-655, 2018.

[17] C. J. Pretorius, J. R. Tate, U. Wilgen, L. Cullen, and J. P. J. Ungerer, "A critical evaluation of the Beckman Coulter Access hsTnI: analytical performance, reference interval and concordance," Clinical Biochemistry, vol. 55, pp. 49-55, 2018.

[18] I. L. Gunsolus, A. S. Jaffe, A. Sexter et al., "Sex-specific 99th percentiles derived from the AACC Universal Sample Bank for the Roche Gen 5 cTnT assay: comorbidities and statistical methods influence derivation of reference limits," Clinical Biochemistry, vol. 50, no. 18, pp. 1073-1077, 2017.

[19] C. Chenevier-Gobeaux, É. Bonnefoy-Cudraz, S. Charpentier et al., "High-sensitivity cardiac troponin assays: answers to frequently asked questions," Archives of Cardiovascular Diseases, vol. 108, no. 2, pp. 132-149, 2015.

[20] G. L. Ackland, T. E. F. Abbott, T. F. Jones et al., "Early elevation in plasma high-sensitivity troponin $\mathrm{T}$ and morbidity after elective noncardiac surgery: prospective multicentre observational cohort study," British Journal of Anaesthesia, vol. 124, no. 5, pp. 535-543, 2020.

[21] S. Agewall, E. Giannitsis, T. Jernberg, and H. Katus, "Troponin elevation in coronary vs. non-coronary disease," European Heart Journal, vol. 32, no. 4, pp. 404-411, 2011.

[22] J. T. Saunders, V. Nambi, J. A. De Lemos et al., "Cardiac troponin $\mathrm{T}$ measured by a highly sensitive assay predicts coronary heart disease, heart failure, and mortality in the atherosclerosis risk in Communities study," Circulation, vol. 123, no. 13, pp. 1367-1376, 2011.

[23] P. Welsh, D. Preiss, C. Hayward et al., "Cardiac troponin T and troponin I in the general population," Circulation, vol. 139, no. 24, pp. 2754-2764, 2019.

[24] M. O. Gore, S. L. Seliger, C. R. Defilippi et al., "Age- and sexdependent upper reference limits for the high-sensitivity cardiac troponin T assay," Journal of the American College of Cardiology, vol. 63, no. 14, pp. 1441-1448, 2014.

[25] A. Aimo, J. L. Januzzi Jr., G. Vergaro et al., "Prognostic value of high-sensitivity troponin $\mathrm{T}$ in chronic heart failure," Circulation, vol. 137, no. 3, pp. 286-297, 2018.

[26] K. Kurz, E. Giannitsis, J. Zehelein, and H. A. Katus, "Highly sensitive cardiac troponin T values remain constant after brief exercise- or pharmacologic-induced reversible myocardial ischemia," Clinical Chemistry, vol. 54, no. 7, pp. 1234-1238, 2008.

[27] P. G. Camici and F. Crea, "Coronary microvascular dysfunction," European Heart Journal, vol. 35, no. 17, pp. 830840, 2014.

[28] O. Bergmann, R. D. Bhardwaj, S. Bernard et al., "Evidence for cardiomyocyte renewal in humans," Science, vol. 324, no. 5923 , pp. $98-102,2009$.
[29] P. Anversa and A. Leri, "Innate regeneration in the aging heart: healing from within," Mayo Clinic Proceedings, vol. 88, no. 8, pp. 871-883, 2013.

[30] Y. Sato, H. Fujiwara, and Y. Takatsu, "Cardiac troponin and heart failure in the era of high-sensitivity assays," Journal of Cardiology, vol. 60, no. 3, pp. 160-167, 2012.

[31] N. A. Marston, M. P. Bonaca, P. Jarolim et al., "Clinical application of high-sensitivity troponin testing in the atherosclerotic cardiovascular disease framework of the current cholesterol guidelines," JAMA Cardiology, vol. 5, no. 11, pp. 1255-1262, 2020. 